\title{
ON A CLASS OF EXACT LOCALLY CONFORMAL COSYMLECTIC MANIFOLDS
}

\author{
I. MIHAI and L. VERSTRAELEN \\ Department Wiskunde, Katholieke Universiteit Leuven \\ Celestijnenlaan 200 B, B - 3000 Leuven, Belgium \\ R. ROSCA \\ 59 Avenue Emile Zola \\ 75015 Paris, France \\ (Received May 6, 1993 and in revised form June 19y4)
}

\begin{abstract}
An almost cosymplectic manifold $M$ is a $(2 m+1)$-dimensional oriented Riemannian manifold endowed with a 2 -form $\Omega$ of rank $2 m$, a 1 -form $\eta$ such that $\Omega^{\prime \prime} \wedge \eta \neq 0$ and a vector field $\xi$ satısfying $i_{\xi} \Omega=0$ and $\eta(\xi)=1$. Partıcular cases were considered in [3] and [6].

Let $(M, g)$ be an odd dimensional oriented Riemannian manifold carrying a globally defined vector field $T$ such that the Riemannian connection is parallel with respect to $T$. It is shown that in this case $M$ is a hyperbolic space form endowed with an exact locally conformal cosymplectic structure. Moreover $T$ defines an infinıtesımal homothety of the connection forms and a relative infinitesimal conformal transformation of the curvature forms.

The existence of a structure conformal vector field $C$ on $M$ is proved and their properties are investigated. In the last section, we study the geometry of the tangent bundle of an exact locally conformal cosymplectic manifold.
\end{abstract}

KEY WORDS AND PHRASES: Locally conformal cosymplectic manifold, $T$-parallel connection, infinitesimal homothety, infinitesimal conformal transformation, Hamiltonian vector field, tangent bundle, Liouville vector field, complete lift, mechanical system.

1991 AMS SUBJECT CLASSIFICATION CODES: 53C15, 53C05, 53C20, 58A15, 58A30.

\section{INTRODUCTION}

In the last decade a series of papers have been devoted to almost cosymplectic manifolds $M(\Omega, \eta, \xi, g)$. As is well known, an almost cosymplectic manifold $M$ is an odd dimensional (say $2 m+1$ ) oriented manifold, where the triple $(\Omega, \eta, \xi)$ of tensor fields is

i) a 2-form $\Omega$ of rank $2 m$

ii) a 1-form $\eta$ such that $\Omega^{n} \wedge \eta \neq 0$

iii) a vector field (called the Reeb vector field) such that $i_{\xi} \Omega=0$ and $\eta(\xi)=1$.

One has the following more studied cases:

$1^{\circ} \Omega$ and $\eta$ are both closed forms. Then $M$ is called a cosymplectic manifold.

$2^{\circ} d \eta=0, d \Omega=2 \eta \wedge \Omega$. Then $M$ is called a Kenmotsu manifold.

$3^{\circ} d \eta=\omega \wedge \eta, d \Omega=2 \omega \wedge \Omega$. Then $M$ is called a locally conformal cosymplectic manifold (see [3],[16]). In this case $\omega$ and its dual vector $T=b^{-1}(\omega)$ with respect to $g$ is called the Lee form (or characteristic form) and Lee vector field respectively.

In the present paper we consider an almost cosymplectic manifold $M(\Omega, \eta, \xi, g)$ carrying a globally defined vector field $T$ whose dual form $b(T)$ is denoted by $\omega$.

Next denote by $0=\operatorname{vect}\left\{e_{A} ; A=0,1, \ldots, 2 m\right\}$ an orthonormal vector basis on $M$ and by $\left\{\theta_{B}^{A}\right\}$ the associated connection forms. If the connection forms satisfy

$$
\theta_{B}^{A}=\left\langle T, e_{B} \wedge e_{A}\right\rangle ; \wedge \text { is the wedge product, }
$$


then one has

$$
\nabla_{l} e_{A}=0
$$

Therefore we agree to say that $M$ is structured by a $T$-parallel connection. In this condition the following significative fact emerges: the almost cosymplectic structure $1 \times S p(2 m, \mathbf{R})$ of $M$ moves to an exact locally contormal cosymplectic structure $1 \times \operatorname{Sp}(2 m, \mathbf{R})$ (abbreviated exact L.C.C.), having $T$ (resp. $\omega=-d f / f$ ) as Lee vector field (resp. Lee form).

Moreover any such a manifold $M$ is a space form of curvature $-2 c$ and $f$ is the energy function corresponding to a Hamiltonian vector field associated with $T$ (in the sense of [3]). If $\theta$ (resp. $\Theta$ ) represents the indexless (or generic) connection torms (resp. curvature forms) of $M$, then $T$ defines an infinitesimal homothety of $\theta$, t.e. $L_{l} \theta=2 c \theta$, and a relative infinitesimal $T$ conformal transformation of $\Theta$ and $\Omega$, ..e.

$$
d\left(L_{T} \Theta\right)=2 c \omega \wedge \Theta, \quad d\left(L_{T} \Omega\right)=2 c \omega \wedge \Omega .
$$

In Section 3 the existence of a structure conformal vector field $C$ on $M$ is proved, i.e.

$$
\nabla_{L} C=\lambda Z+g(Z, T) C-g(Z, C) T ; \lambda \in C^{\infty} M, \quad Z \in \Gamma(T M) .
$$

Moreover $C$ is a divergence conformal vector field, i.e. grad (div $C$ ) is a concurrent vector field and it defines an infinitesimal conformal transformation of:

1) the conformal cosymplectic form $\Omega$, i.e. $L_{c} \Omega=\rho \Omega, \rho=2 \lambda$;

ii) the dual forms $\omega^{A}$, i.e. $L_{C} \omega^{A}=\frac{\rho}{2} \omega^{A}$;

iii) the curvature forms $\Theta_{B}^{4}$, i.e. $L_{C} \Theta_{B}^{A}=\rho \Theta_{B}^{A}$;

iv) all the $(2 q+1)$-forms $\alpha_{q}=b(C) \wedge \Omega^{q}$, i.e. $L_{C} \alpha_{q}=(1+q) \rho \alpha_{q}$;

v) all the functions $g(C, Z)$, i.e. $L_{C} g(C, Z)=\rho g(C, Z), Z \in \Gamma(T M)$.

In the last section, we discuss some properties of the tangent bundle manifold $T M$ having as basis the exact (L.C.C.)-manifold $M$. Denote by $V, \gamma$ and $v$ the Liouville vector field ([13]), the Liouville 1 -form and the Liouville function respectively, on $T M$.

The following properties are proved:

i) the complete lift $\Omega^{c}$ of $\Omega$ is a $d^{-\omega}$-exact 2 -form ( $d^{\omega}$ is the cohomological operator [11]) and is homogeneous of class 1 , i.e.

$$
L_{V} \Omega^{c}=\Omega^{c}
$$

ii) $\quad \gamma$ satisfies $d^{-1} \gamma=\psi$ and $\psi$ is a Finslerian form, i.e.

$$
L_{v} \psi=\psi, \quad i_{v} \psi=0
$$

(iv denotes the vertical differentiation operator [11]);

iii) the vertical lift $T^{\mathrm{v}}$ of $T$ defines an infinitesimal automorphism of $\psi$, i.e. $L T^{\mathrm{v}} \psi=0$;

iv) the function $r=f v$ and the 2-form $f \psi$ define a regular mechanical system $\mathcal{M}([13])$ having $r$ as kinetic energy and $f \psi$ as canonical symplectic (exact) form.

\section{PRELIMINARIES}

Let $(M, g)$ be a Riemannian $C^{\infty}$-manifold and let $\nabla$ be the covariant differential operator with respect to the metric tensor $g$. Assume that $M$ is oriented and $\nabla$ is a Levi-Civita connection. Let $\Gamma(T M)=\chi(M)$ and $b: T M \rightarrow T^{*} M$ be the set of sections of the tangent bundle $T M$ and the musical isomorphism ([18]) defined by $g$, respectively. Following [18] we set 
and notice that elements of $A^{q}(M, T M)$ are vector valued $q$-forms $(q \leq \operatorname{dim} M)$.

Denote by $d^{\mathrm{v}}: A^{\mathrm{q}}(M, T M) \rightarrow A^{q+1}(M, T M)$ the exterior covariant derivative operator with respect to $\nabla$. It should be noticed that generally $d^{v^{\prime}}=d^{v} \circ d^{v} \neq 0$ unlike $d^{2}=d \circ d=0$. If $p \in M$, then the vector valued 1 -torm $d p \in A^{1}(M, T M)$ is the canonical vector valued 1 -form of $M([5])$ and since $\nabla$ is symmetric one has $d^{\mathrm{v}}(d p)=0$. The operator

$$
d^{(\omega)}=d+e(\omega)
$$

acting on $\Lambda M$, where $e(\omega)$ means the exterior product by the closed 1 -form $\omega$, is called the cohomological operator $([11])$. One has

$$
d^{\omega} O d^{\omega}=0 .
$$

Any form $u \in \Lambda M$ such that $d^{\prime \prime \prime} u=0$ is said to be $d^{\omega}$-closed and if $\omega$ is an exact form, then $u$ is sald to be a $d^{\text {"' }}$-exact form. Any vector field $Z \in \Gamma(T M)$ such that

$$
d^{\nabla}(\nabla Z)=\nabla^{2} Z=\pi \wedge d p \in A^{2}(M, T M)
$$

for some 1 -form $\pi$, is said to be an exterior concurrent vector field ([17]). The form $\pi$ which is called the concurrence form is given by

$$
\pi=\lambda b(Z) ; \quad \lambda \in C^{\infty} M .
$$

A non flat manifold of dimension $m>2$ is an elliptic or hyperbolic space-form if and only if every vector field on $M$ is an exterior concurrent one ([17]). On the tangent bundle manifold $T M, d_{v}$ and $i$, define the vertical differentiation and the vertical derivation operators respectively ([7]). $d_{v}$ is an antiderivation of degree 1 on $\Lambda(T M)$ and $i_{v}$ is a derivation of degree 0 on $\nabla(T M)$.

In an $n$-dimensional Riemannian manifold $M$, denote by

$$
\mathbf{O}=\operatorname{vect}\left\{e_{A} ; A=1, \ldots, n\right\}
$$

a local field of orthonormal frames and let

$$
\mathbf{O}^{*}=\operatorname{covect}\left\{\omega^{A} ; A=1, \ldots, n\right\}
$$

be its associated coframe.

The soldering form $d p$ is expressed by

$$
d p=\omega^{A} \otimes e_{A}
$$

and E. Cartan's structure equations written indexless manner are

$$
\begin{aligned}
& \nabla e=\theta \otimes e \\
& d \omega=-\theta \wedge \omega \\
& d \theta=-\theta \wedge \theta+\Theta
\end{aligned}
$$

Any vector field $T$ such that

$$
\nabla T=s d p+u \otimes T, \quad u \in \Lambda^{1} M
$$

is called a torse forming (K. Yano [20]). If $d u=0$, then $T$ is a closed torse forming, which implies that $T$ is an exterior concurrent vector field, and if $u=0$, then $T$ is a concurrent vector field ([22]).

Let now $W$ be any conformal vector field on $M$ (i.e. the conformal version of Killing's equations). As is well known, $W$ satisfies

$$
L_{W} g=\rho g \quad \text { or } \quad g\left(\nabla_{Z} W, Z^{\prime}\right)+g\left(\nabla_{Z}, W, Z\right)=\rho g\left(Z, Z^{\prime}\right)
$$

where the conformal scalar $\rho$ is defined by

$$
\rho=\frac{2}{n}(\operatorname{div} W) .
$$

We recall some basic formulas which we shall use in the following sections. 


$$
\begin{gathered}
L_{k} b(Z)=\rho b(Z)+b[W, Z] \quad \text { (Orsted lemma) } \\
L_{w} K=(n-1) \Delta \rho-K \rho \\
2 L_{k} S\left(Z, Z^{\prime}\right)=(\Delta) \rho g\left(Z, Z^{\prime}\right)-(n-2) \quad\left(\operatorname{Hess} \nabla^{\rho}\right)\left(Z, Z^{\prime}\right) .
\end{gathered}
$$

In the above equations $L_{k}, K, \Delta$ and $S$ denote the Lie derivative with respect to $W$, the scalar curvature of $M$, the Laplacian and the Ricci tensor field of $\nabla$, respectively. One has

$$
\left(\operatorname{Hess}_{\vee} \rho\right)\left(Z, Z^{\prime}\right)=g\left(Z, H_{\rho} Z^{\prime}\right), \quad H_{\rho} Z^{\prime}=\nabla_{Z^{\prime}}(\operatorname{grad} \rho)
$$

(see also $|2|)$.

\section{EXACT LOCALLY CONFORMAL COSYMPLECTIC MANIFOLDS}

Let $(M, g)$ be a $(2 m+1)$-dimensional oriented Riemannian $C^{\infty}$-manifold and let $T=\sum_{A=0}^{2 m} t^{\wedge} e_{A}$ and $\omega=b(T)$ be a globally defined vector field on $M$ and its dual form respectively.

Denote by $\mathbf{O}=\operatorname{vect}\left\{e_{A} ; A=0,1, \ldots, 2 m\right\}$ (resp. $\theta_{B}^{A}$ ) a local field of orthonormal frames on $\boldsymbol{M}$ (resp. the associated connection forms). Recall that the vectorial wedge product $\wedge$ is defined by

$$
\begin{gathered}
(X \wedge Y) Z=g(Y, Z) X-g(X, Z) Y ; Z \in \Gamma(T M) \\
\text { i.e. } \quad X \wedge Y=b(Y) \otimes X-b(X) \otimes Y .
\end{gathered}
$$

Assume now that all the connection forms $\theta$ satisfy

$$
\theta_{B}^{A}=\left\langle T, e_{B} \wedge e_{A}\right\rangle
$$

Then by the structure equations (1.6), it follows at once

$$
\theta_{B}^{A}=t^{B} \omega^{A}-t^{A} \omega^{B}
$$

It should be noticed that if $\theta$ satisfy (2.2) one has $\theta(T)=0$ and the above equation shows that all the connection forms $\theta$ are relations of integral invariance for the vector field $T$ (in the sense of $A$. Lichnerowicz [14]).

Next by the structure equations (1.6) and by (2.2) one obtains

$$
\nabla e_{A}=t^{A} d p-\omega^{A} \otimes T
$$

and the above equation implies

$$
\nabla_{T} e_{\mathrm{A}}=0 .
$$

From (2.4) the following significative fact emerges: all the vectors of the $\mathbf{O}$-basis are $T$-parallel. Therefore we agree to say that the Riemannian manifold under consideration is structured by a $T$-parallel connection (abr. T.P.).

Further again by (2.2) one derives by the structure equations (1.7)

$$
d \omega^{\wedge}=\omega \wedge \omega^{\wedge} ; \quad \omega=b(T)=t^{\wedge} \omega^{\wedge}
$$

which by a simple argument implies that the dual form $\omega$ of $T$ is closed, i.e.

$$
d \omega=0 \text {. }
$$

Thus in terms of $d^{\omega}$-cohomology, (2.5) may be written as

$$
d^{-\omega} \omega^{A}=0
$$

and $\mathbf{O}^{*}=\left\{\omega^{A}\right\}$ is defined as a $d^{-\omega}$-closed covector basis.

Now for reasons which will soon appear, we set

$$
\omega^{0}=\eta, \quad e_{0}=\xi
$$


and consider on $M$ the globally defined 2 -torm $\Omega$ of rank $2 m$ given by

$$
\Omega=\Sigma \omega^{\prime \prime} \wedge \omega^{a^{*} ;} \quad a=1, \ldots, m ; \quad a^{*}=a+m .
$$

Then since $\Omega^{\prime \prime} \wedge \eta \neq 0, i_{\S} \Omega=0$, one may say that the triple $(\Omega, \eta, \xi)$ defines an almost cosymplectic structure $1 \times S p(2 m, \mathbf{R})$ having $\xi$ as Reeb's vector tield.

Next takıng the exterıor differentıal of $\Omega$ a short calculation gives with the help of (2.5)

$$
d \Omega=2 \omega \wedge \Omega \Leftrightarrow d^{-2(\omega)} \Omega=0
$$

and by $(2.5)$ we may write

$$
d \eta=\omega \wedge \eta \Leftrightarrow d^{-11} \eta=0 .
$$

We conclude that any odd dimensional Riemannien manifold $M$ structured by a $T$-parallel connection is endowed with a locally conformal cosymplectic structure $1 \times C S p(2 n, \mathbf{R})$ (abr. L.C.C.). We notice that the vector field $T$ (resp. the 1 -form $\omega=b(T)$ ) is the Lee vector field (resp. the Lee form) of this structure.

Moreover since $\omega=t^{4} \omega^{4}$, then by a simple argument it follows on behalf of $(2.5)$ that one may set

$$
d t^{A}=f \omega^{A} ; \quad f \in C^{\infty} M
$$

which by exterior differentiation gives instantly

$$
\omega=-d f / f \text {. }
$$

Therefore since $\omega$ is an exact form, it follows on behalf of a known terminology, that the manifold $M$ under consideration is an exact (L.C.C.)-manifold. We agree to call $f$ the distinguished scalar field associated with the exact (L.C.C.)-structure.

Now taking the covariant differential of $T$ one finds by (2.3) and (2.12)

$$
\nabla T=(f+2 l) d p-\omega \otimes T
$$

where we have set

$$
g(T, T)=2 l
$$

Using (2.12) and (2.15), we have

$$
d l=\int \omega \Rightarrow l+f=c=\text { const } \neq 0
$$

and (2.14) becomes

$$
\nabla T=(l+c) d p-\omega \otimes T .
$$

Hence, by (1.9) and (2.6) $T$ is a closed torse forming and consequently an exterior concurrent (abr. E.C.)-vector field.

Operating now on $\nabla e_{A}$ and $\nabla T$ by the exterior covariant derivative operator $d^{\nabla}$, one gets by (2.12) and (2.16)

$$
\begin{aligned}
& d^{\nabla}\left(\nabla e_{A}\right)=\nabla^{2} e_{A}=2 c \omega^{A} \wedge d p \\
& d^{\nabla}(\nabla T)=\nabla^{2} T=2 c \omega \wedge d p .
\end{aligned}
$$

From the above equations it is seen that any vector field $Z$ on $M$ is E.C. with constant conformal scalar $2 c$. Therefore on behalf of the general properties of E.C.-vector fields ([17]), we may state the following striking property: the exact L.C.C.-manifold $M(\Omega, \eta, \xi)$ under discussion is a space-form of curvature $-2 c$.

As a consequence, it follows that the curvature forms $\Theta$ are expressed by

$$
\Theta_{B}^{A}=-2 c \omega^{A} \wedge \omega^{B}
$$

Next taking the exterior differential of the forms $\Theta$, one quickly finds by

$$
d \Theta_{B}^{A}=2 \omega \wedge \Theta_{B}^{A} \Leftrightarrow d^{-2 \omega} \Theta_{B}^{A}=0
$$


which shows that all the curvature forms $\Theta$ are $d^{-210}$-exact.

On the other hand taking the Lie derivatives of the covectors $\omega^{1}$ of $\mathbf{O}^{*}$ one derives by (2.12) and (2.16)

$$
L_{l} \omega^{A}=(l+c) \omega^{A}-t^{A} \omega
$$

Theretore since $L$, satısfies Leibniz rule one deduces by $(2.20)$

$$
L_{I} \Theta_{B}^{4}=2(l+c) \Theta_{B}^{4}+2 c \cdot \theta_{B}^{4} \wedge \omega
$$

Similarly, we obtain

$$
d \Theta_{B}^{4}=2 \int \omega^{B} \Lambda \omega^{4}+\omega \Lambda \theta_{B}^{A}
$$

Clearly by (2.12) one has $L_{l} t^{A}=f t^{A}$ and with the help of (2.22) we deduce

$$
L_{I} \theta_{B}^{A}=2 c \theta_{B}^{A} .
$$

Accordingly by the above equations we may say that the Lie vector field $T$ defines on infinitesimal homothety of all the connection forms $\theta$.

Taking now the exterior differential of the equations (2.23), a standard calculation gives

$$
d\left(L_{T} \Theta_{B}^{A}\right)=8 c \omega \wedge \Theta_{B}^{A}
$$

which proves that $T$ defines a relative infinitesimal conformal transformation ([19]) of the curvature forms.

let $\mu: T M \rightarrow T^{*} M, \mu(Z)=i_{L} \Omega$ be the bundle isomorphism defined by $\Omega$ and set $\bar{\omega}=\mu(T)$, i.e.

$$
\bar{\omega}=i_{T} \Omega=\sum_{a=1}^{m}\left(t^{a} \omega^{a^{*}}-t^{a^{*}} \omega^{a}\right)
$$

for the dual form of $T$ with respect to $\Omega$. By (2.5) and (2.12) an easy calculation gives

$$
d \bar{\omega}=2 f \Omega+\omega \wedge \bar{\omega}
$$

and by $(2.10)$ and $(2.13)$ one gets

$$
L_{T} \Omega=2(l+c) \Omega+\bar{\omega} \wedge \omega
$$

and consequently by $(2.28)$ it follows

$$
d\left(L_{T} \Omega\right)=2 c \omega \wedge \Omega .
$$

Hence as for the curvature forms $\Theta, T$ defines a relative conformal transformation of the structure 2-form $\Omega$.

Consider now the vector valued 1 -form

$$
F=\omega^{a} \otimes e_{a^{*}}-\omega^{a^{*}} \otimes e_{a} \in A^{1}(M, T M) .
$$

If $Z$ is any vector field, a simple calculation gives

$$
\langle F, Z\rangle=Z^{a} e_{a^{*}}-Z^{a^{*}} e_{a}=\bar{Z}
$$

which implies

$$
g\left(Z, Z^{\prime}\right)+g\left(Z, \bar{Z}^{\prime}\right)=0, \quad Z, Z^{\prime} \in \Gamma(T M)
$$

and $\langle F, d p\rangle=2 \Omega$.

On the other hand since $\bar{\omega}(T)=0$ one gets by $(2.27)$

$$
L_{T} \bar{\omega}=2 c \bar{\omega}
$$

that is $T$ defines an infinitesimal homothety of $\bar{\omega}=(\mu \circ b) T$.

Next by (2.12) and (2.13) one easily gets 


$$
i_{\bar{l}} \Omega=\frac{d f}{f}-\frac{1}{f} \xi(f) \eta
$$

Therefore by reference to $|3|$ one may call $\bar{T}$ the cosymplectic Hamiltonian vector field of $M$ and the distınguished scalar $\int$ turns out to be the energy function corresponding to $\bar{T}$.

Moreover by $(2.35)$ one derives

$$
L_{\bar{l}} \Omega=\eta(\bar{T}) \eta \wedge\left(\omega \Rightarrow d\left(L_{\bar{I}} \Omega\right)=0\right.
$$

whıch shows that $\bar{T}$ detınes a relative infinıtesımal automorphısm (R. Abraham [1]) of $\Omega$.

Summing up, we state the following

THEOREM. Let $M$ be a $(2 m+1)$-dimensional Riemannian manifold and let $T$ be a globally defined vector field on $M$. If $M$ is structured by a $T$-parallel connection, then $M$ is endowed with an exact locally conformal cosymplectic structure $1 \times C S p(2 m, \mathbf{R})$, having $T$ (resp. $\omega=b(T)$ ) as Lee vector (resp. Lee torm) and any such an $M$ is a space-form of curvature $-2 c$.

Moreover one has the following properties:

i) $T$ defines an infinitesimal homothety of the connection forms $\theta$ and of the 1 -form $\mu(T)$, i.e.

$$
L_{T} \theta=2 c \theta, \quad L_{T} \mu(T)=2 c \mu(T)
$$

ii) $T$ defines a relative infinitesimal conformal transformation of the curvature forms $\Theta$ and of the structure 2 -form $\Omega$, i.e.

$$
d\left(L_{T} \Theta\right)=8 c \omega \wedge \Theta, \quad d\left(L_{I} \Omega\right)=2 c \omega \wedge \Omega
$$

iii) the vector field $\bar{T}=\left(b^{-1} \circ \mu\right) T$ (resp. $f$ ) is the cosymplectic Hamiltonian associated with the $1 \times \operatorname{CSp}(2 m, \mathbf{R})$-structure of $M$ (resp. its corresponding energy function) and $\bar{T}$ defines a relative infinitesimal automorphism of $\Omega$.

Let now $\Phi: M \rightarrow \bar{M}$ be a conformal diffeomorphism (abr. C.D.) that is

$$
\Phi: g \rightarrow e^{2 \sigma} g=\bar{g} ; \quad \sigma \in C^{\infty} M .
$$

One also say that $g$ and $\tilde{g}$ are conformally equivalent metrics and setting $e^{2 \sigma}=v^{2}$, we agree to call the function $v$ the argument of the C.D.

As is shown one has for $Z, Z^{\prime} \in \Gamma(T M)$

$$
\tilde{\nabla} Z=\nabla Z+b(\operatorname{grad} \sigma) \otimes Z-b(Z) \otimes \operatorname{grad} \sigma+g(Z, \operatorname{grad} \sigma) d p
$$

or equivalently

$$
\tilde{\nabla}_{Z} Z=\nabla_{Z} Z+Z^{\prime}(\sigma) Z+Z(\sigma) Z^{\prime}-g\left(Z, Z^{\prime}\right) \text { grado }
$$

and if $K$ and $\tilde{K}$ denote the scalar curvature of $M$ and $\tilde{M}$ respectively then one has ([8])

$$
\tilde{K}=e^{-2 \sigma}\left\{K+2(n-1)(n-2)\|\operatorname{grad} \sigma\|^{2}\right\}
$$

$(n=\operatorname{dim} M)$.

If $M$ is an exact (L.C.C.)--manifold, its Ricci tensor field $S$ satisfies

$$
S\left(Z, Z^{\prime}\right)=-4 m c g\left(Z, Z^{\prime}\right) ; \quad Z, Z^{\prime} \in \Gamma(T M)
$$

and the scalar curvature $K$ is given by

$$
K=-4 m(2 m+1) c
$$

Perform now a conformal transformation of $M$ having as argument $e^{\sigma}$ the energy function $f$. It is obvious that

$$
(2.42) d \sigma=d f / f=-\omega .
$$

Then we have grad $\sigma=-T$, which implies 


$$
\Delta \mathrm{s}=\operatorname{div} T=(2 m+1) c+(2 m-1) l .
$$

Hence by (2.41) and (2.43) we derive at once from (2.39), $\tilde{K}=0$, that is $\tilde{M}$ is a flat manifold. We notice that this fact is in accordance with the known

PROPOSITION. A Riemannian manıfold of constant curvature is conformally flat, provided $n \geq 3$.

Using (2.37) one may prove that all vectors $\bar{e}_{A}$ are parallel (the connection forms $\tilde{\theta}_{B}^{A}$ vanish, i.e. $\tilde{\nabla}$ Is a tlat connection). Thus we have

PROPOSITION. It $M$ is an exact (L.C.C.)-manifold with metric tensor $g$ and energy function $f$, then the metric $f^{2} g$ is flat.

\section{STRUCTURE CONFORMAL VECTOR FIELDS ON AN EXACT (L.C.C.)-MANIFOLD}

In consequence of some conformal properties induced by the $T$-parallel connection which structures $M(\Omega, \eta, \xi, g)$ we are naturally led to see if the manifold $M$ under consideration carries a structure conformal vector field $C$ in the sense of [6], [15]. Therefore the covariant differential of $C$ is expressed by

$$
\nabla C=\lambda d p+C \wedge T=\lambda d p+\omega \otimes C-\alpha \otimes T ; \quad \lambda \in C^{\infty} M, \quad \alpha=b(C) .
$$

Put

$$
C=C^{A} e_{A} \Rightarrow b(C)=\alpha=C^{A} \omega^{A}
$$

and $s=g(C, T)$. Then by $(2.3)$ and (3.1) one quickly gets

$$
\begin{aligned}
d C^{A} & =(\lambda-s) \omega^{A}+C^{A} \omega \\
d \alpha & =2 \omega \wedge \alpha \Rightarrow d^{-2 \omega} \alpha=0 .
\end{aligned}
$$

Next since $d s=\langle\nabla C, T\rangle+\langle\nabla T, C\rangle$, a short calculation gives

$$
\begin{aligned}
& d s=\lambda \omega-(l-c) \alpha \\
& d s=d \lambda
\end{aligned}
$$

By (3.4), (3.5) and (3.6) it is seen that the existence of $C$ is assured by an exterior differential system $\Sigma$ whose characteristic numbers are

$$
r=3, s_{0}=2, s_{1}=1 .
$$

Then $\Sigma$ is in involution in the sense of $E$. Cartan (i.e. $r=s_{c}+s_{1}$ ). Accordingly one may say that the existence of $C$ depends on 2 arbitrary functions of one argument (E. Cartan's test). The conformal scalar $\rho$ associated with $C\left(L_{C} g=\rho g\right)$ is given by

$$
\rho=2 \lambda \text {. }
$$

By a short calculation one has

$$
[C, T]=-\lambda T-(l-c) C ; \quad[\quad]: \text { Lie bracket }
$$

and from $(3.5)$ it follows

$$
L_{C} \omega=d s=\lambda \omega-(l-c) \alpha .
$$

This equation matches by Orsted's lemma (1.12) the expression of $[C, T]$.

On the other hand since $C$ is necessarily an E. C. vector field ( $M$ is a space-form), then operating (3.1) by $d^{\nabla}$ and taking account of (3.4) and (3.5), one derives

$$
d^{\nabla}(\nabla C)=\nabla^{2} C=2 c \alpha \wedge d p .
$$

The above equation is coherent with the properties obtained in Section 2.

Setting now

$$
\bar{\alpha}=\iota_{C} \Omega=\Sigma\left(C^{a} \omega^{a^{*}}-C^{a^{*}} \omega^{a}\right)
$$


one gets by (3.4) and (2.5)

$$
d \vec{\alpha}=2(\lambda-s) \Omega+2 \omega \wedge \bar{\alpha}
$$

and one follows

$$
L_{c} \Omega=\rho \Omega .
$$

Hence (3.13) reveals that $C$ defines an infinitesimal conformal transformation (abr. I.C.T.) of the contormal cosymplectic form $\Omega$.

By similar methods, one gets by (2.5), (2.24), (2.20) and (2.21)

$$
L_{C} \omega^{A}=\frac{\rho}{2} \omega^{A}, \quad L_{C} \theta_{B}^{A}=\frac{\rho}{2} \theta_{B}^{A}, \quad L_{C} \Theta_{B}^{A}=\rho \Theta_{B}^{A} .
$$

Therefore one may say that $C$ defines an I.C.T. of the exact (L.C.C.)-structure of $M$.

Moreover let $L$ be the operator of type (1.1) on forms defined by $S$. Goldberg ([8]), that is $L u=u \wedge \Omega ; u \in \Lambda^{1} M$, and consider on $M$ the $(2 q+1)$-forms

$$
L^{q} \alpha=\alpha_{q}=\alpha \wedge \Omega^{q} .
$$

Since by Orsted's lemma one has

$$
L_{C} \alpha=\rho \alpha
$$

then by (3.13) and a standard calculation one derives

$$
L_{C} \alpha_{q}=(q+1) \rho \alpha_{q} .
$$

Hence $C$ defines an (I.C.T.) of all the $(2 q+1)$-forms $\alpha_{q}$.

Next since $C$ is a conformal vector field, then as is known (see (1.11)) one has

$$
d \iota v=(\rho / 2)(2 m+1)
$$

and since $\rho=2 \lambda$ it follows by (3.5) and (3.6) that

$$
\operatorname{grad} \rho=\rho T+2(c-l) C .
$$

Further by (2.16) and taking account of (2.14) and (3.1) it is easily deduced

$$
\nabla \operatorname{grad} \rho=2 c \rho d p .
$$

Thus one may state the following relevant property: the gradient of the associated scalar $\rho$ of $C$ is a concurrent vector field (K. Yano and B. Y. Chen [22]). We agree to call a conformal vector field such that the gradient of its conformal scalar $\rho$ is a concurrent vector field, a divergence conformal vector field. Such a situation occurs also when studying conformal vector fields on Lorentzian P.S. manifolds (see I. Mihai and R. Rosca [15]).

On the other hand from (2.14) one derives

$$
\operatorname{div} T=(2 m-1) l+(2 m+1) c
$$

and since $\operatorname{div} C=(2 m+1) \lambda$, one gets on behalf of $(3.20)$

$$
\Delta \rho=-\operatorname{div}(\operatorname{grad} \rho)=-2(2 m+1) c \rho
$$

which shows that $\rho$ is an eigenfunction of $\Delta$.

$C$ being an E.C. vector field satisfying (3.10), one has ([17])

$$
S(C, Z)=-4 m c g(C, Z), \quad Z \in \Gamma(T M)
$$

where $S$ denotes the Ricci tensor field of $\nabla$.

Now making use of (1.14) and carrying out the calculations, one finds by (3.19) and (3.22)

$$
L_{C} g(C, Z)=\rho g(C, Z) .
$$

Hence the vector field $C$ defines an I.C.T. of all the functions $g(C, Z)$, where $Z \in \Gamma(T M)$.

Concuding, we have proved the following

THEOREM. Let $M$ be the exact (L.C.C.) manifold defined in Section 2 and $C$ a structure conformal vector field on $M$ (which existence is proved), i.e. 


$$
\nabla C=\frac{\rho}{2} d p+C \wedge T ; \quad L_{C} g=\rho g
$$

Then $C$ is a divergence contormal vector tield (i.e. grad(div $C$ ) is a concurrent vector field) and it defines the followıng intınitesımal conformal transtormations

$$
\begin{gathered}
L_{C} \Omega=\rho \Omega, \quad L_{c} \omega^{\prime}=\frac{\rho}{2} \omega^{\prime}, \quad L_{C} \theta_{b}^{\prime}=\frac{\rho}{2} \theta_{B}^{4} \\
L_{c} \Theta_{b}^{\prime}=\rho \Theta_{b}^{\prime}, \quad L_{c}\left(\alpha_{q}=(1+q) \rho \alpha_{4}, \quad L_{C} g(C, Z)=\rho g(C, Z)(Z \in \Gamma(T M)\right.
\end{gathered}
$$

where $\Omega, \omega^{4}, \theta_{B}^{4}, \Theta_{B}^{1}$ and $\alpha_{a}=b(C) \wedge \Omega^{4}$ are the conformal symplectic 2 -form, the dual forms, the connection forms, the curvature torms and the $(2 q+1)$-torms defined by the $(1,1)$-operator $L$, respectively on $M$.

\section{GEOMETRY OF THE TANGENT BUNDLE OF AN EXACT (L.C.C.)-MANIFOLD}

Let now $T M$ be the tangent bundle manifold having the exact (L.C.C.)-manifold $M$ discussed in Section 2 as a basis.

Denote by $V\left(v^{A}\right)(A=0,1, \ldots, 2 m)$ the Liouville vector field (or the canonical vector field [7]). Accordingly we may consider the set $B^{*}=\left\{\omega^{A}, d v^{A}\right\}$ as an adapted cobasis in $T M$. Following Godbillon ([7]) we denote by $d_{v}$ and $i_{v}$ the vertical differentiation and the vertical derivative operators with respect to $B^{*}$, respectively $\left(d_{v}\right.$ is an antiderivation of degree 1 on $\Lambda(T M)$ and $i_{v}$ is a derivation of degree 0 on $\Lambda(T M))$. Let $T_{s}^{r} M$ be the set of all tensor fields of type $(r, s)$ on $M$.

In general as is known $([23])$ the vertical and complete lifts are linear mappings of $T_{s}^{r} M$ into $T_{s}^{r}(T M)$ and one has

$$
\left(T_{1} \otimes T_{2}\right)^{c}=T_{1}^{v} \otimes T_{2}^{c}+T_{1}^{c} \otimes T_{2}^{v}
$$

In the case under discussion we may define the complete lift $\Omega^{c}$ of the structure 2-form $\Omega$ of $M$ by the 2-form of rank $4 m$ on $T M$

$$
\Omega^{c}=\Sigma\left(d \mathrm{v}^{a} \wedge \omega^{a^{*}}+\omega^{a} \wedge d \mathrm{v}^{a^{*}}\right), \quad a=1, \ldots, m ; \quad a^{*}=a+m .
$$

On the other hand since the Liouville vector field $V$ is expressed by

$$
V=\Sigma v^{A} \frac{\partial}{\partial v^{A}}
$$

then as is known the basic 1 -form

$$
\gamma=\Sigma v^{A} \omega^{A}
$$

is called the Liouville form (see also [13]).

Taking now the exterior differential of $\Omega^{c}$ one finds by (2.5)

$$
d \Omega^{c}=\omega \wedge \Omega^{c} \Leftrightarrow d^{-\omega} \Omega^{c}=0
$$

which shows that $\Omega^{c}$ is similarly as $\Omega$ a $d^{-\omega}$-exact form. We recall that in general conformal properties are not preserved by complete lifts ([23]).

One has

$$
i_{V} \Omega^{c}=\Sigma\left(\mathrm{v}^{a} \omega^{a^{*}}-\mathrm{v}^{a^{*}} \omega^{a}\right)
$$

which implies $\omega(V)=0$ and so by (4.5) and (4.6) one gets

$$
L_{V} \Omega^{c}=\Omega^{c}
$$

Accordingly on behalf of a known definition ([13]), the above equation shows that $\Omega^{c}$ is of class 1, a homogeneous form on TM. Taking now the exterior differential of the Liouville form $\gamma$ defined by (4.4), one gets at once by (2.5)

$$
d \gamma=\omega \wedge \gamma+\psi \Leftrightarrow d^{-\omega} \gamma=\psi
$$


where we have set

$$
\psi=\sum d v^{A} \wedge \omega^{A}
$$

From (4.8) and (1.2) one obtains instantly

$$
d^{-\prime \prime \prime} \psi \prime=() \Leftrightarrow d \psi=\omega \wedge \psi .
$$

Since clearly the 2-form $\psi$ is of maximal rank, we agree to call $\psi$ the canonical conformal symplectic form of $M$. Noticing that one has

$$
\iota_{V} \psi=\gamma, \quad \omega(V)=0
$$

which implies

$$
L_{V} \psi=\psi
$$

Hence $\psi$ is as $\Omega^{\prime}$ a homogeneous of class 1,2 -form.

Next making use of the vertical operator $i_{v}$ defined by $i_{v} \lambda=0, i_{v} d v^{A}=\omega^{A}, i_{v} \omega^{A}=0\left(\lambda \in C^{\infty} M\right)$ one quickly finds by (4.9)

$$
i_{v} \psi=0
$$

and the above equation together with (4.12) proves that $\psi$ is a Finslerian form ([7]).

We recall that the vertical lift $Z^{\mathrm{v}}([23])$ of a vector field $Z \in \Gamma(T M)$ with components $Z^{A}$ in $M$, has as components

$$
Z^{\mathrm{v}}=\left(\begin{array}{l}
0 \\
Z^{A}
\end{array}\right)=Z^{A} \frac{\partial}{\partial v^{A}}
$$

Hence in the case under consideration one has

$$
T^{\mathrm{v}}=\Sigma t^{A} \frac{\partial}{\partial \mathrm{v}^{A}} ; \quad A=0,1, \ldots, 2 m
$$

and by (4.9) one gets

$$
i_{T^{\nu}} \psi=\omega
$$

Therefore by (4.10) one derives

$$
L_{T^{\nu}} \psi=0
$$

and one may say that $T^{\vee}$ defines an infinitesimal automorphism of $\psi$.

Finally we set

$$
r=f v
$$

where

$$
\mathrm{v}=\frac{1}{2} \Sigma\left(v^{A}\right)^{2}
$$

denotes the Liouville function on $M$ ([9]).

Operating on $r$ by the vertical differentiation operator $d_{v}([7])$ one gets

$$
d_{v} r=f \sum_{A} v^{A} \omega^{A}=f \mu
$$

and taking the exterior differential of (4.19) we obtain by (2.13) and (4.9)

$$
d\left(d_{v} r\right)=f \Sigma d v^{A} \wedge \omega^{A}=f \psi
$$

Next putting $I I=f \psi$ it follows by (2.13)

$$
d I I=0 .
$$

Therefore the exact symplectic form II can be viewed as the canonical symplectic form of the $(4 m+2)$-dimensional manifold $T M([13])$.

Finally by reference to [13] one may consider that the pair $(r, I I)$ defines a regular mechanical system $\mathcal{M}$ (in the sense of Klein [13]) having the scalar $r$ as kinetic energy. 
THEOREM. Let $T M$ be the tangent bundle manitold having as basis the exact (L.C.C.)-manifold $M(\Omega, T, \omega)$ discussed in Section 2. Let $V, \gamma$ and $v$ be the Liouville vector field, the Liouville form and the Liouville function of $T M$, respectively. One has the following properties:

i) the complete lift $\Omega$ on $T M$ of the contormal cosymplectic form $\Omega$ of $M$ is a homogeneous of

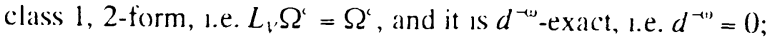

ii) $\gamma$ satisfies $d^{-11} \gamma=\psi \Rightarrow d^{-1} \psi=()$ and $\psi$ is the canonical conformal symplectic form of $T M$ and $\psi$ enjoys also the property to be a Finslerian form;

iii) the vertical lift $T^{\vee}$ of $T$ defines an infinitesimal automorphism of $\psi$, i.e. $L_{T^{v}} \psi=0$;

Iv) $\quad r=f v$ and $f \psi$ define a regular mechanical system on $T M$ having $r$ as kinetic energy and $f \psi$ as canonical symplectic form (where $f$ is the energy function of $M$ ).

\section{REFERENCES}

[1] ABRAHAM, R. Foundations of Mechanics, W. A. Benjamin Inc., New York (1967).

[2] BRANSON, T. Conformally covariant equations of differential forms, Comm. Partial Diff. Equations, 7 (1982), 393-431.

[3] CHINEA, D., DE LEON, M. and MORRERO, J. C. Locally conformal cosymplectic manifolds and time-dependent Hamiltonian systems, Comm. Math. Univ. Carolinae, 32 (1991), 383-387.

[4] DATTA, D. K. Exterior recurrent forms in a manifold, Tensor N.S., 36 (1982), 115-120.

[5] DIEUdOnNÉ, J. Treaties on Analysis, Vol. 4, Academic Press, New York (1974).

[6] DONATO, S. and ROSCA, R. Structure conformal vector fields on almost paracontact manifolds with parallel structure vector, Osterreiche Akademie des Wissenschaften, Wien, 198 (1989), 201-209.

[7] GODBILlON, C. P. Géométrie Differentielle et Mécanique Analitique, Hermann, Paris (1969).

[8] GOLDBERG, S. Curvature and Homology, Academic Press, New York (1962).

[9] GOLDBERG, V. V. and ROSCA, R. Pseudo-Sasakian manifolds endowed with a contact conformal connection, Inernat. J. Math. and Math. Sci., 9 (1986), 733-747.

[10] GOLDBERG, V. V. and ROSCA, R. Foliate conformal Kählerian manifolds, Rend. Sem. Mat. Messina, Serie II, Vol. I (1991), 105-122.

[11] GUEDIRA, F. and LICHNEROWICZ, A. Géométrie des algébres de Lie locales de Kirilov, J. Math. Pures Appl., 63 (1984), 407-494.

[12] KERMOTSU, K. A class of almost contact Riemannian manifolds, Tohoku Math. J., 24 (1972), 93-103.

[13] KLEIN, I. Espaces variationels et mécanique, Ann. Inst. Fourier, 12 (1962), 1-124.

[14] LICHNEROWICZ, A. Les relations intégrals d'invariance et leura applications a la dynamique, Bull. Sci. Math., 70 (1946), 82-95.

[15] MIHAI, I. and ROSCA, R. On Lorentisian P-Sasakian manifolds, Classical Analysis, World Scientific Publ., Singapore (1992), 155-169.

[16] OLCSAK, Z. and ROSCA, R. Normal locally conformal almost cosymplectic manifolds, Publicationes Math. (Debrecen), 39 (1991), 315-323.

[17] PETROVIC, M., ROSCA, R. and VERSTRAELEN, L. On exterior concurrent vector fields I. Some general results, Socehow J. Math., 15 (1989), 179-187.

[18] POOR, W. A. Differential Geometric Structures, McGraw Hill Book Co., New York (1981).

[19] ROSCA, R. On some infinitesimal transformations in Riemannian and pseudo-Riemannian manifolds (Preprint).

[20] YANO, K. On the torse-forming directions in Riemannian spaces, Proc. Imp. Acad., Tokyo, 20 (1944), 340-345.

[21] YANO, K. Integral Formulas in Riemannian Geometry, M. Dekker, New York (1970).

[22] YANO, K. and CHEN, B. Y. On the concurrent vector fields of immersed manifolds, Kodai Math. Sem. Rep., 23 (1971), 343-350.

[23] YANO, K. and ISHIHARA, S. Differential Geometry of Tangent and Cotangent Bundles, M. Dekker, New York (1973). 


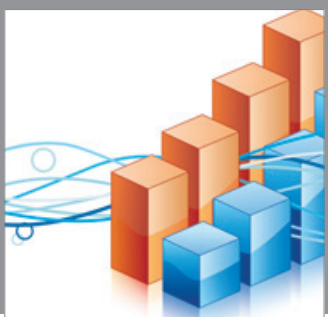

Advances in

Operations Research

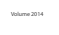

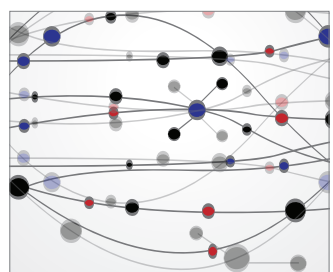

\section{The Scientific} World Journal
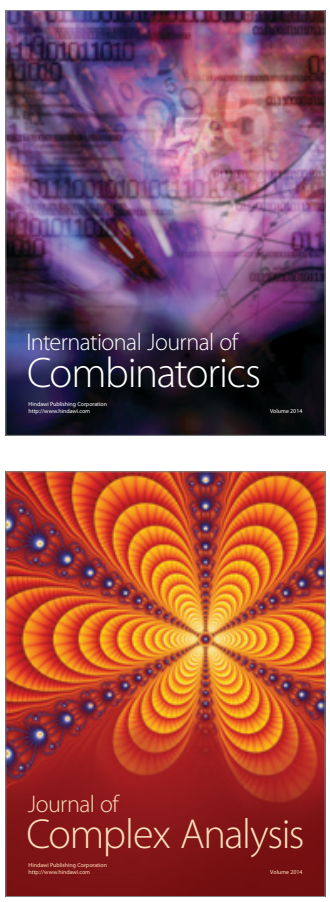

International Journal of

Mathematics and

Mathematical

Sciences
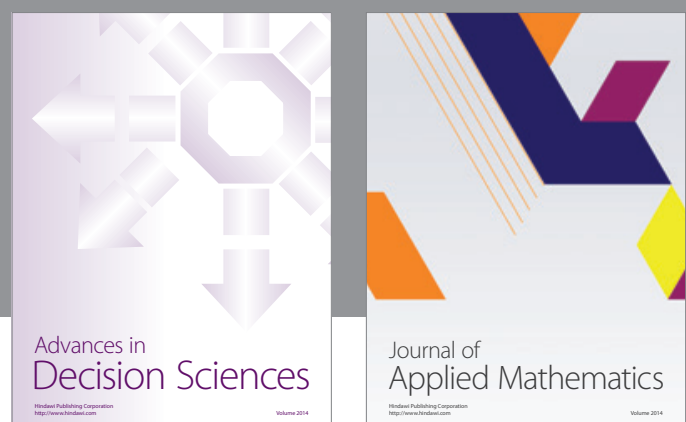

Journal of

Applied Mathematics
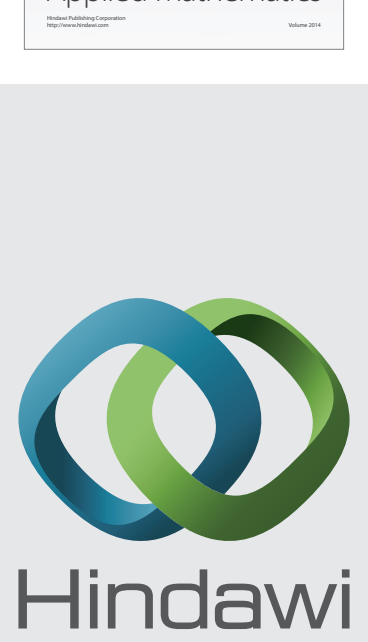

Submit your manuscripts at http://www.hindawi.com
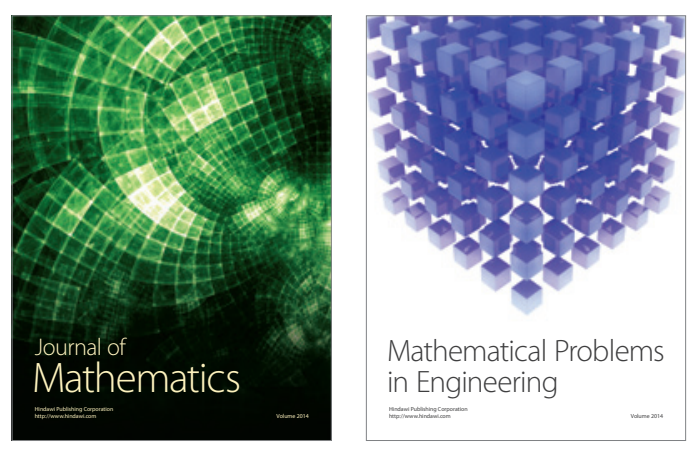

Mathematical Problems in Engineering
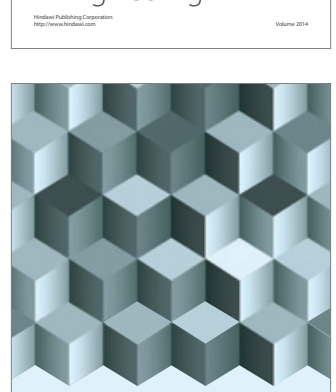

Journal of

Function Spaces
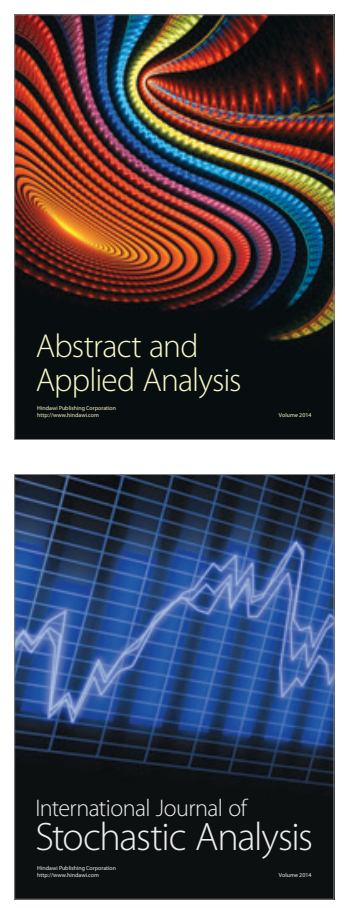

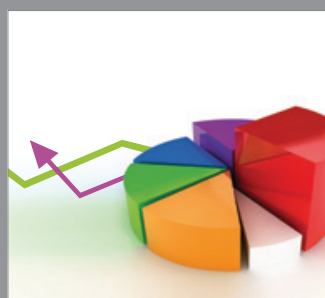

ournal of

Probability and Statistics

Promensencen
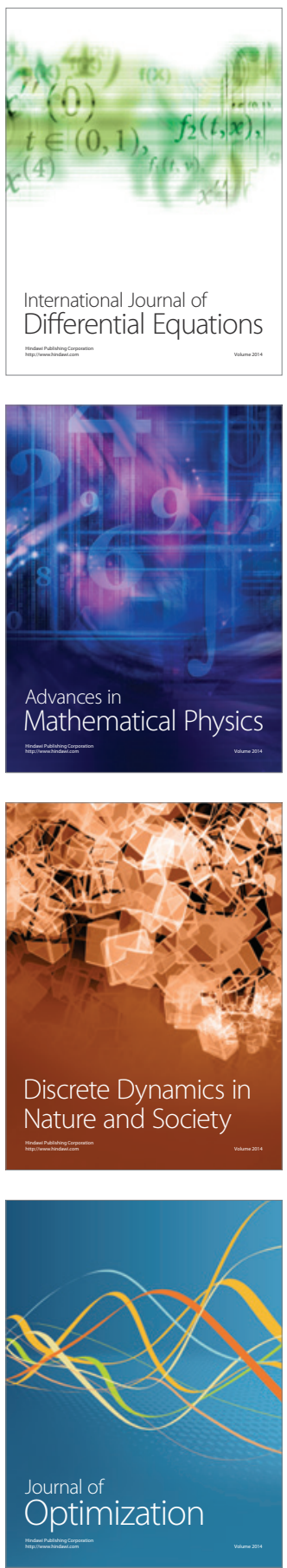